\title{
Ultra-fast escape of a deformable jet-propelled body
}

\author{
G. D. Weymouth ${ }^{1, \dagger}$ and M. S. Triantafyllou ${ }^{2}$ \\ ${ }^{1}$ Southampton Marine and Maritime Institute, University of Southampton, Southampton, SO17 1BJ, UK \\ ${ }^{2}$ Center for Ocean Engineering, Massachusetts Institute of Technology, 77 Massachusetts Avenue, \\ Cambridge, MA 02139, USA
}

(Received 5 December 2012; revised 18 January 2013; accepted 23 January 2013)

In this work a cephalopod-like deformable body that fills an internal cavity with fluid and expels it to propel an escape manoeuvre, while undergoing a drastic external shape change through shrinking, is shown to employ viscous as well as mainly inviscid hydrodynamic mechanisms to power an impressively fast start. First, we show that recovery of added-mass energy enables a shrinking rocket in a dense inviscid flow to achieve greater escape speed than an identical rocket in a vacuum. Next, we extend the shrinking body results of Weymouth \& Triantafyllou (J. Fluid Mech., vol. 702, 2012, pp. 470-487) to three-dimensional bodies and show that three hydrodynamic mechanisms must be combined to achieve rapid escape performance in a viscous fluid: added-mass energy recovery; flow separation elimination; and an optimized energy storage and recovery. In particular, we show that the mechanism of separation elimination achieved through rapid body shrinking, coordinated with the mechanism of recovering the initially imparted added-mass energy, is critical to achieving a high escape speed. Hence a flexible, collapsing body can be vastly superior to a rigid-shell jet-propelled body.

Key words: biological fluid dynamics, boundary layer control, drag reduction

\section{Introduction}

In this paper we consider the mechanics of rapid acceleration of a flexible body in a viscous fluid. The inspiration comes from the reported performance of cephalopods, i.e. the octopus, the squid and the cuttle fish, but we simplify the problem to study the basic hydrodynamic mechanisms at work rather than analyse the performance of a specific animal: we consider a flexible body that refills a cavity with fluid, hence hyperinflating its external shape to a spherical form, and then expels the fluid rapidly to form a propelling jet, while causing its shape to shrink, taking the form of a prolate spheroid with decreasing radial dimension.

Deformable bodies offer intriguing possibilities of flow control, because their added mass varies with their changing shape allowing significant and rapid variations in the fluid force and the energy exchange between body and fluid. Added-mass energy

$\dagger$ Email addresses for correspondence: weymouth@mit.edu, G.D.Weymouth@soton.ac.uk 
transfer and recovery is shown for a deformable fluid in an inviscid fluid by Saffman (1967) who shows that, as a result, a wake-less propulsion is possible. Daniel (1984) considered the unsteady aquatic locomotion of animals, arguing that in periodic motion of deformable bodies the average added-mass force is zero and hence does not affect overall propulsion. Kanso et al. (2005) also considered motion in potential flow where a body controls its motion by actively changing its shape variables. In the experimental work of Childress, Vanderberghe \& Zhang (2006), a body with flexible surface was tested in oscillatory flow, with a variable frontal area exposed to the flow. The flapper-like body could suspend itself against gravity by taking advantage of a change in added mass between its upstroke and downstroke. Also, Spagnolie \& Shelley (2009) conducted numerical simulations varying the frontal area of a twodimensional cylinder against an oscillatory oncoming flow. Propulsive forces were obtained by actively controlling the phase difference between the shape change and the fluid oscillation. Childress, Spagnolie \& Tokieda (2011) considered propulsion through simultaneous change in shape (and hence added mass) and change in the distribution of the mass the body, resulting in what they termed recoil locomotion. Hence, in these studies, a change in object area or shape was responsible for the net force on the body, as unequal levels of kinetic energy are imparted by the body to the flow in opposing stroke planes.

The model system in this study is inspired by cephalopods, which have a highly developed jet propulsion system and, although they lack a skeleton, can match the performance of vertebrates. Squids, for example, swim as well as many fishes using jet propulsion, while small squids, such as the larvae of Loligo vulgaris, can achieve bursts of 25 body lengths per s (Gosline \& DeMont 1985). Water is taken into a compression chamber, the mantle cavity, through a wide inlet, the mantle aperture, and then squeezed out through a narrow funnel that can vector in any desired direction (Packard 1969). Their mantle contains muscles that are circumferentially arranged to pressurize the mantle cavity by contraction and hence help expel the water to form a jet (Anderson, Wuinn \& deMont 2001).

It is particularly interesting to study the rapid escape response, where an animal employs its full power over a short period of time. Marine animals have to confront the large reactive forces of a heavy liquid and must avoid flow separation that can induce additional, large resistive forces. While the escape responses of fish have been studied in detail experimentally and theoretically (Domenici \& Blake 1997; Domenici, Blagburn \& Bacon 2011a,b; Gazzola, van Rees \& Koumoutsakos 2012), the escape trajectories of cephalopods has attracted less attention.

Squids escaping predators employ a mode called escape jetting (Williamson 1965), whereby the squid first hyperinflates, increasing its lateral dimension by up to $30 \%$, and then contracts its mantle at full power, while keeping its fins wrapped closely against the body (Gosline \& DeMont 1985). Neumeister et al. (2000) show that squid (Loligo opalescens), ranging from 95 to $125 \mathrm{~mm}$ in mantle length, when stimulated by a visual flash employ escape jetting, to travel 4 mantle lengths within $700 \mathrm{~ms}$ with a maximum velocity of 10 mantle lengths per s, reached at $\sim 400 \mathrm{~ms}$.

The escape response in the octopus is similar, although there are differences as well (Gosline \& DeMont 1985): water is brought into the mantle cavity and then expelled quickly through the funnel (Wells 1990), with the mantle forward and the legs tightly trailing behind the body (Forsythe \& Hanlon 1988). Longitudinal muscles prevent the elongation of the mantle as the radial muscles pressurize the mantle cavity. Maximum jetting speeds are reported to be 3.29 body lengths per s for mantle length of $45 \mathrm{~mm}$ in Huffard (2006). In addition, medusoid jetting may occur, when the octopus opens 
and then closes its arms and arm crown like an umbrella to aid jet propulsion (Huffard 2006).

Interestingly, the relatively bluff initial shape of cephalopods does not prevent them from achieving impressive velocities and bursts of acceleration. Unlike man-made rigid-hull rockets, cephalopods can also derive thrust as well as control their local flow field through the change in the size and shape of their bodies during jetting. Also, of particular relevance are the results of Weymouth \& Triantafyllou (2012) who studied the effect of rapidly shrinking the diameter of two-dimensional circular cylinders moving steadily in viscous flow. It was shown that such size reduction in a viscous flow may enable recovery of added-mass energy from the fluid, depending on the kinematics and form of body shrinking employed. At one extreme is a 'melting' cylinder, defined as a body which undergoes progressively a phase change from solid to fluid, starting from the outer layers and progressing to the inner layers. This case allows no energy recovery and large-scale vortices form which entrain the differential from the original to the final added-mass energy. A contrasting case is that of a rapidly collapsing cylinder, where there is no mass flux through the body boundary. In this case the potential-like flow energy can be largely recovered, while the initial vorticity in the boundary layer is partially annihilated by opposite-sign vorticity generated during the shrinking. The result is that no significant vorticity is shed and hence no wake forms. In this paper we first show that these results hold for three-dimensional bodies as well, and then focus on the case of a deflating body, as it provides a mechanism for separation elimination and energy recovery.

To study this mechanism of unsteady flow control and propulsion, in this work we consider the model problem of a deflating prolate spheroid, which starts from rest and accelerates by ejecting the fluid contained in its cavity to form a propelling jet. First, in $\S 2$, we consider the governing equations for self-propelled rockets in vacuo and in a fluid, and demonstrate that a shrinking rocket in inviscid flow can outperform a rocket in a vacuum via added-mass energy recovery. As viscous effects cannot be ignored in the real system, we next review our numerical methodology for the simulation of general viscous flows in $\S 3$, and then present a series of prescribed speed tests in $\S 4$. These preliminary tests allow us to isolate the effect of body shrinking, particularly in preventing flow separation, from the other effects in the self-propelled system. In particular, a shape-change number is defined in $\S 4.3$, which parameterizes the ability of a deflating body to reduce separation, and thereby reduce drag. Finally, in $\S 5$, we address the principal problem under study, that of a self-propelled body shrinking and expelling fluid to propel itself. We show that an initially bluff-body rocket which deflates can suppress separation, enabling added mass recovery, and achieving nearly double the final speed of a rigid streamlined body.

\section{Energetics of jet-propelled motion}

First we contrast the governing equations and simplified analytic solutions for three cases of jet-propelled systems, sketched in figure 1, which we will call 'rockets' since they expel mass to produce a propulsive force:

(a) a rocket in vacuo and without external forcing;

(b) a rigid rocket submerged in a dense fluid; and

(c) a deformable rocket, which deflates as it propels itself within a dense fluid.

Radically different results are obtained for each case, highlighting the effects of the fluid on rocket propulsion and non-obvious advantages of deflation. 
(a)

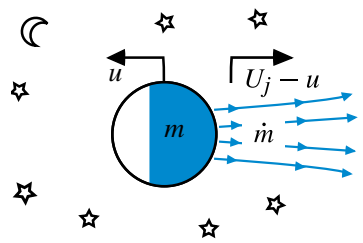

(b)

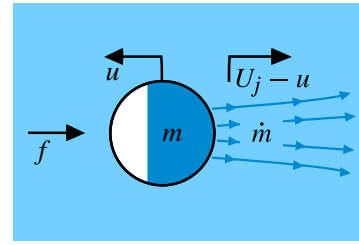

(c)

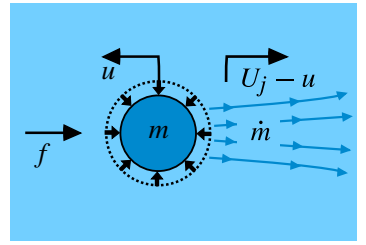

FIGURE 1. (Colour online) Sketches of the three rocket systems considered in $\S 2$ : $(a)$ rocket without forcing; $(b)$ rigid rocket in fluid; $(c)$ shrinking rocket in fluid. In all cases the mass $m$ (dark grey, blue online) is ejected as propellant at rate $\dot{m}$ and velocity $U_{j}$ relative to the body speed $u$. In a fluid (light grey), the rocket experiences fluid forcing $f$, which strongly influences the dynamics. The rigid rocket $(b)$ reduces its average density as it empties of propellant but maintains constant volume, while the shrinking rocket $(c)$ maintains constant density as its shape shrinks.

\subsection{Rocket without external forcing}

Consider a body of initial mass $m_{o}$ which expels its mass at a rate $\dot{m}$ forming a jet with velocity $U_{j}$, relative to a system fixed on the body, to produce the force that drives the motion. The velocity of the body $u$ is obtained by the equation of motion:

$$
m \dot{u}=-\dot{m} U_{j}+f,
$$

where $f$ is an external force, $\dot{m}$ is negative (loss of body mass), and $U_{j}$ is positive.

If the rocket is operating in a light fluid relative to its density, or in vacuo as in figure $1(a)$, the external forces may be neglected. If we further assume the jet velocity is kept constant, then the increment of velocity of the body, $\Delta u$, is obtained simply as:

$$
\frac{\Delta u}{U_{j}}=-\ln \left(\frac{m_{f}}{m_{o}}\right),
$$

where $m_{f}$ is the final mass of the rocket, after expelling the propellant. Hence, the speed increase simply depends on the ratio of the initial to the final mass, reaching arbitrarily large values as $m_{f} / m_{o} \rightarrow 0$. If the jet speed is variable, it can be shown that it is advantageous to ramp up the speed with time, because as the mass diminishes near the end of the manoeuvre, the resulting higher thrust provides much higher acceleration and hence final speed. It is clear, however, that the size and shape of this simple rocket do not have an impact on its effectiveness in a vacuum.

\subsection{Rocket in a fluid}

If the body is travelling through a fluid with density $\rho$, which is comparable to the density of the body, then the external fluid forcing $f$ on the body cannot be neglected. The equation of motion now includes a reactive force from the fluid, in the form of a term that involves the fluid added mass $m_{a}$, and a resistive (drag) force $f_{d}$ :

$$
m \dot{u}+\frac{\partial}{\partial t}\left(m_{a} u\right)=-\dot{m} U_{j}-f_{d} .
$$

Unlike the previous case, the size and shape of the body are now important in determining the propulsive performance, because they influence the added-mass force and the drag force. In this section and the next we will focus on the inviscid (added mass) effects and assume the drag force to be negligible. 
To demonstrate the basic properties of (2.3), we consider the case of figure 1(b): a rigid spherical body with initial density $\rho$, whose diameter $D$ and added mass

$$
m_{a}=\frac{1}{12} \pi \rho D^{3}
$$

are fixed. As with the previous section, this rocket propels itself by expelling mass down to a final mass $m_{f}$, and as a result the final average density of the rocket is less than that of the fluid.

If we use a constant jet speed as before, we find:

$$
\frac{\Delta u}{U_{j}}=-\ln \left(\frac{2 m_{f} / m_{o}+1}{3}\right) .
$$

Comparing (2.5) and (2.2), we find that even when neglecting fluid resistance, the performance of a rigid rocket in a fluid is tightly constrained. As $m_{f} / m_{o} \rightarrow 0$ the increase in velocity cannot exceed $\approx 1.1 U_{j}$. The fluid adds inertia to the system, and acts as an additional payload, greatly reducing the rocket performance relative to the vacuum case.

\subsection{Fast shrinking rocket}

Next we consider a body which shrinks as it expels the fluid propellant, as in the case of the cephalopods. Again, as sketched in figure 1(c), we assume a spherical shape, with initial diameter $D_{o}$, but as the diameter reduces so does the added mass. Assuming a constant jet velocity as before, the velocity increment for the shrinking rocket is:

$$
\frac{\Delta u}{U_{j}}=2\left(\frac{D_{o}}{D_{f}}-1\right)=2\left(\left[\frac{m_{f}}{m_{o}}\right]^{-1 / 3}-1\right),
$$

where $D_{f}$ is the final diameter of the sphere.

We note that allowing the body to shrink has re-enabled the rocket to achieve arbitrarily large velocity as $m_{f} / m_{o} \rightarrow 0$, as in the case of a rocket in vacuum. More surprisingly, the performance of a shrinking rocket in an inviscid fluid is better than in a vacuum for $m_{f} / m_{o}<0.1$. For example, for $m_{f} / m_{o}=0.05$ there is a $16 \%$ increase in speed relative to motion in a vacuum, while for $m_{f} / m_{o}=0.01$ the speed increase is close to $60 \%$.

This ultra-fast performance in a fluid cannot be explained simply by the vanishing added-mass force. Instead the speed-up is achieved because there is energy 'stored' in the fluid at the early phase of the motion through the added-mass term, which is then recovered at the final stage of the manoeuvre. This is beneficial because near the end of the manoeuvre the body has shed a major part of its mass, increasing the effectiveness of the applied thrust.

However, in a viscous fluid the drag force will substantially reduce the final value of the velocity, particularly for the case of a spherical body, where flow separation can be significant and the resulting drag forces large. Hence, it makes a significant difference whether the drag force is caused by skin friction alone, when the resistive force is small, versus the case when flow separation takes place, when the drag coefficient can increase very substantially. As a result, a sphere is expected to develop substantial drag force and hence will be in general inferior to a streamlined ellipsoid. We study these cases next through numerical simulation of the viscous fluid equations. 


\section{Numerical methodology}

To obtain detailed quantitative results for a flexible body in a viscous fluid, a set of three-dimensional viscous simulations of translating and shrinking spheroids were preformed. These simulations use the boundary data immersion method (BDIM), a robust immersed boundary method suitable for dynamically deforming bodies with prescribed kinematics.

The numerical details of the simulation method follow those described in Weymouth et al. (2006) and Weymouth \& Yue (2011). The full Navier-Stokes equations and the solid-body dynamic equations are integrated over the solid and fluid domains with a kernel of finite support $\epsilon$. This has the effect of blending the equations smoothly over the solid/fluid interface. The integrated equations are valid over the complete domain and allow general solid-body dynamics and solid/fluid interfacial conditions to be applied. Previous problems examined with the method include ship flows and flexible wavemaker flows (Weymouth et al. 2006), solid-body free-surface impact and cavity formation (Weymouth \& Yue 2011), as well as shedding of vorticity from a rapidly displaced foil (Wibawa et al. 2012) and a melting and collapsing circular cylinder (Weymouth \& Triantafyllou 2012).

The analytic BDIM equations are discretized using a second-order finite-volume method in space and Heun's explicit second-order method in time. A body-centred computational domain is used which is $8 D_{o}$ on each side, where $D_{o}$ is the initial diameter of the body. All cases use the no-slip tangential boundary conditions on the solid/fluid interface. The no-penetration condition is used for the body's normal velocity, except for the melting body which is discussed separately in $\S 4.1$, and across the jet exit which is given a uniform velocity profile with magnitude $U_{j}$, relative to the rocket. Pressure conditions are applied on the sidewalls and a convection exit condition is used to allow fluid to enter and leave the domain freely, reducing the impact of these numerical boundaries. The computational grid has more than $300 \times 10^{6}$ grid points with spacing $\Delta x / D_{o}=1 / 125$ near the body surface and geometric expansion of the spacing in the far field. The implicit large-eddy simulation method of Margolin, Rider \& Grinstein (2006) is used to model the subgrid-scale effects. Convergence studies on the results in $\S \S 4.1,4.3$ and 5 run using grids with $\Delta x / D_{o}=1 / 100$ and $\Delta x / D_{o}=1 / 80$ showed no qualitative difference and the quantitative differences between the two finest grids were less than $2 \%$ for all cases. As such, we have high confidence in the accuracy of these viscous three-dimensional simulations.

\section{Simulation results for a towed deformable body}

Next we address through simulation the problem of a deformable body undergoing rapid size reduction within a viscous fluid. First, we conducted simulations on a 'towed' rather than a self-propelled flexible body; hence the body is forced to move at a prescribed speed. This allows us to isolate the effect of body shrinking, particularly in preventing flow separation, from other effects such as the interaction of the flow around the body with the propelling jet.

\subsection{Study of collapsing and melting spheres}

An initial set of tests was performed to investigate the nature and balance of forcing described in (2.3) with the simplification that the body is towed at prescribed speed instead of being self-propelled.

In Weymouth \& Triantafyllou (2012) we studied the effects of rapid shrinking in two-dimensional cylinders moving within a viscous fluid, showing that a collapsing 
cylinder can recover, to a great extent, the potential-flow-like energy, while the initial vorticity in the boundary layer is annihilated by opposite-sign vorticity generated during the shrinking; as a result, no vorticity is shed to form a wake. In contrast, a body which undergoes rapid but orderly 'melting' of the outer layers of the cylinder, in the sense of a simple (non-thermodynamic) phase change from solid to fluid, allows no such recovery, and large-scale vortices are shed entraining the original added-mass energy. While the focus in Weymouth \& Triantafyllou (2012) was on the latter case, since it allows the rapid development of forces through this global vorticity shedding, the focus here is on the first case, of a collapsing cylinder, with particular attention paid to the elimination of separation.

Hence, we first conducted a set of simulations similar to those in Weymouth \& Triantafyllou (2012), but extending them to the case of a three-dimensional body. In these towed tests, the body is first accelerated to full speed $U$ over a very short distance, equal to $0.05 D_{o}$, and then the speed is kept constant throughout the run. In this way, we can isolate the effects and forces caused by the body deformation so as to compare them to constant-shape results. The Reynolds number for these tests is $R e=1000$, based on $U$ and $D_{o}$, again chosen to match the previous study. We considered three different configurations:

(i) towing of a rigid sphere, first with diameter $D_{o}$ and then with diameter $0.8 D_{o}$;

(ii) a 'melting' sphere whose diameter is reduced from $D_{o}$ to $0.8 D_{o}$ over a short period of time, $0.1 D_{o} / U$, starting when the sphere has travelled $0.25 D_{o}$, i.e. well before the onset of flow separation;

(iii) a collapsing sphere made to shrink at a prescribed rate by applying an appropriate forcing at its surface so as to reduce its diameter over the same time period and rate as the melting case.

Figure 2 shows the resulting changes to the drag and kinetic energy for each of the three configurations we studied. We find similar results and effects as in the two-dimensional study of Weymouth \& Triantafyllou (2012). We show here only the integrated results of force and kinetic energy, but the processes are nearly identical to those in the two-dimensional case.

(a) Starting with the collapsing sphere, we found, as for the two-dimensional cylinder, that opposite-sign vorticity is generated on the upper and lower half of the body surface, relative to the originally developed vorticity. As this occurs before significant flow separation develops, and hence no vorticity has shed, this new vorticity partially annihilates the opposite-sign vorticity that had developed in the boundary layer during the acceleration phase, and 'resets' the drag and kinetic energy of the flow to that corresponding to a smaller sphere of diameter $0.8 D_{o}$. This is discussed further for a prolate spheroid in the next section. During collapse we note a rapid decrease of the drag force, which changes sign to become a large thrust force, lying outside the frame of figure 2(a). Also, the differential in kinetic energy of the fluid, due to the reduction in body diameter, is recovered through the forces at the surface of the sphere, as shown in figure $2(b)$, where the kinetic energy follows initially the curve of the constant-diameter larger sphere, until collapse starts, when the sink-like flow introduces an apparent large kinetic energy; once collapse ends, the kinetic energy returns to the value corresponding to the constant-diameter smaller sphere, in complete analogy with the results for a cylinder (Weymouth \& Triantafyllou 2012).

(b) The 'melting' sphere results are not central to this paper but are shown here to contrast them with the collapsing sphere results, and for completeness, in order 

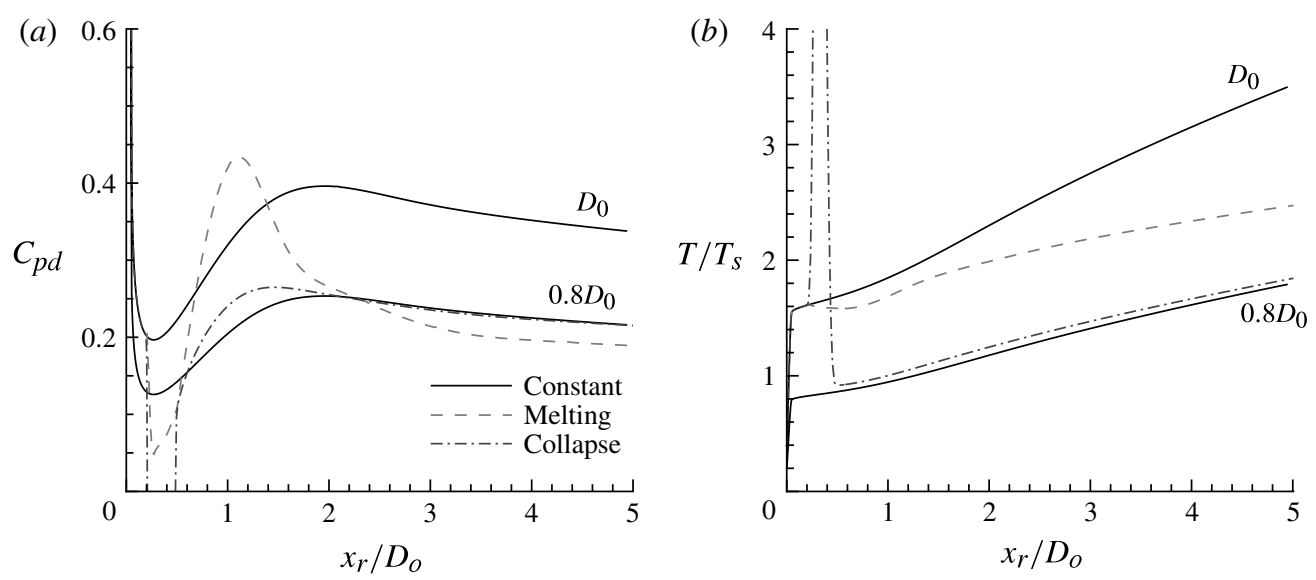

Figure 2. (a) Pressure force coefficient based on $D_{o}, C_{p}=f_{p} /\left(\rho D_{o}^{2} U^{3} / 2\right)$; and $(b)$ total kinetic energy $T$ scaled by the solid-body kinetic energy $T_{s}=\pi \rho D_{o}^{3} U^{2} / 12$, as a function of the distance travelled by the rear stagnation point of the body, $x_{r} / D_{o}$. Results are for four towed cases: - , constant-diameter sphere cases, run first with diameter $D_{o}$ and then $0.8 D_{o}$; -- , rapidly melting sphere, changing diameter from $D_{o}$ to $0.8 D_{o} ;-\cdot-$, rapidly collapsing sphere with the same size change from $D_{o}$ to $0.8 D_{o}$. The collapsing sphere ultimately recovers the kinetic energy initially imparted to the fluid and resets to the smaller-body drag response.

to establish the close similarity of the three-dimensional with the two-dimensional results. As shown in figure 2, the drag force shows a decrease during the period of melting and then a rapid increase as the shed vorticity forms organized structures entraining the kinetic energy differential, which is lost to the body.

While these results confirm and extend for three-dimensional bodies the earlier results of Weymouth \& Triantafyllou (2012), and provide critical insight into the mechanisms of flow control in deformable bodies, they were derived for a bluff body, a sphere. This requires that all transitions be very rapid, namely the manoeuvre must be completed before the body traverses a distance equal to one diameter, beyond which large-scale separation is unavoidable. The question is whether changing from spherical to a streamlined shape will provide the same mechanisms without the limitations of a sphere. This is what we show in the next section.

\subsection{Study of deformation kinematics on a prolate spheroid}

Next we proceed to study the development of thrust and recovery of fluid kinetic energy in the case of a deflating non-spherical body, which is the focus of this paper. We take the body geometry to be that of a prolate spheroid, to more closely match the streamlined form of engineering structures, as well as that of animals, such as the cephalopods.

When cephalopods hyperinflate their shape in order to escape, their form changes initially to a much more bluff shape than under ordinary swimming conditions. We replicate this herein by assuming that the initial geometry is that of a sphere with diameter $D_{o}$; subsequently, the body is collapsed only in the cross-stream (radial) direction, maintaining its in-line length $L=D_{o}$ constant, as the octopus is also reported to do. Hence, the shape changes smoothly from a sphere to an elongated prolate spheroid. 
(a)

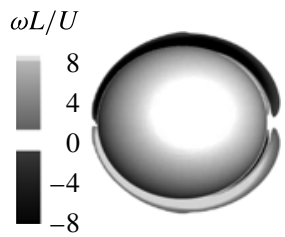

(b)

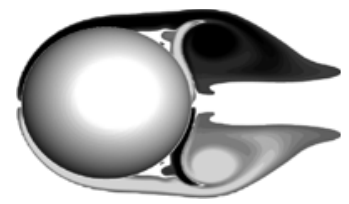

(c)

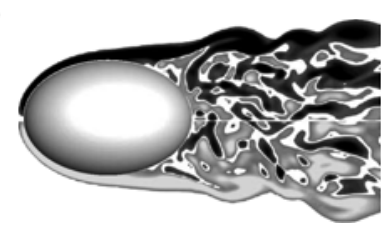

FIGURE 3. Impact of kinematics on the vorticity field $(\omega)$ for a deforming prolate spheroid: (a) abrupt $20 \%$ reduction in diameter over $\Delta t U / L=0.1$, shown at $t U / L=0.3 ;(b, c)$ gradual $80 \%$ reduction in diameter over $\Delta t U / L=25$, shown at $t U / L=3$ in $(b)$ and $t U / L=11$ in (c). Abrupt size change does not cause separation, while a very gradual size change results in separation and formation of a wide wake. Body motion is right-to-left at constant velocity $U$. Contours of vorticity are shown on a plane passing through the body's longitudinal axis of symmetry. $(a, b)$ show the two cases for the same 10:9 aspect ratio. $(c)$ shows the symmetry breaking in the wake for $t U / L>6$.

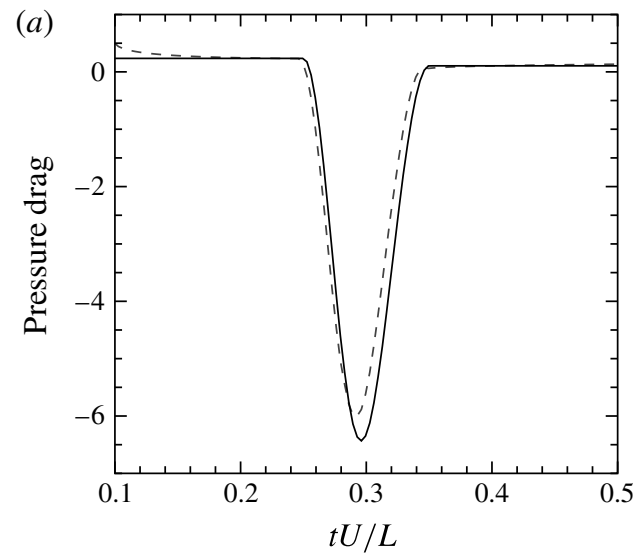

(b)

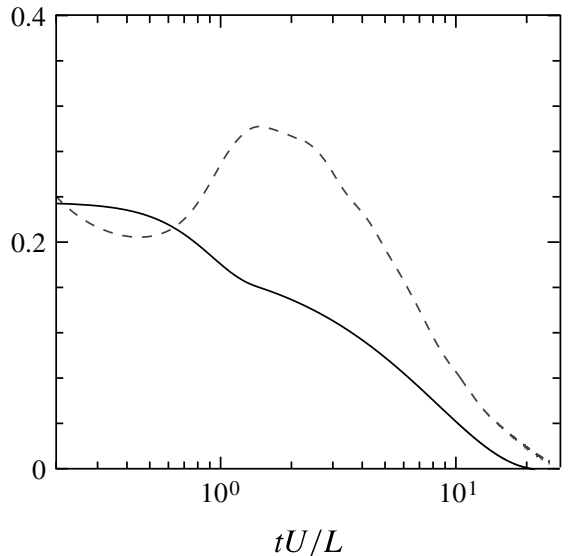

FIGURE 4. Pressure force coefficient acting on the prolate spheroid as function of distance travelled for the two cases shown in figure 3: $(a)$ abrupt $20 \%$ size change; $(b)$ gradual $80 \%$ size change. Simulation results $(--)$ are compared to the theoretical estimate of (4.1) (-). The force is scaled by the initial projected cross-stream area $A_{o}=(\pi / 4) L^{2}$. The negative values in $(a)$ indicate thrust instead of drag:

We find that the ability of the body to recover the energy initially imparted to the fluid, and use it to augment thrust, depends highly on the prescribed kinematics. First, the kinematics are set to match those of the collapsing sphere of $\S 4.1$ : the collapse starts and completes before the spheroid has been towed half a body length, so that its boundary layer has not separated, and the vorticity has not shed to form a reversed flow, as shown in figure $3(a)$; in fact, opposite-sign vorticity annihilates the extra vorticity for the reduced-size body.

In figure 4 the resulting pressure drag forces on the body are compared to a simple analytic model:

$$
f_{p d}=U \frac{\mathrm{d} m_{a}}{\mathrm{~d} t}+\frac{1}{2} c_{p d} \rho U^{2} \pi\left(\frac{D}{2}\right)^{2}
$$


with $c_{p d} \propto(D / L)^{3 / 2}$, which combines the added-mass forcing with a simple drag force model, using a drag coefficient based on the aspect ratio. Equation (2.4) defines the instantaneous added mass for a general prolate spheroid where $D$ is the cross-stream diameter. The measured forces in figure 4(a) show excellent agreement with this simple model, with nearly $95 \%$ of the added-mass energy recovered by the body in viscous flow.

This energy recovery can be achieved only within certain parametric limits: if we use a shrinking velocity much slower than the towing speed, the response changes entirely, as shown in figures $3(b, c)$ and $4(b)$. Because the bluff body is shrinking slowly, the boundary layer has time to separate and the vorticity is shed, forming a wake. As a result, the flow does not resemble a potential flow and the predictions of (2.3) are not quantitatively accurate, and are qualitatively reasonable only after the shed vorticity has moved well downstream.

\subsection{Study of deformation rate on an accelerating prolate spheroid}

Next we conducted a series of tests to establish the direct connection between the rapid shrinking of a deformable body and the ability to inhibit separation - and thus reduce drag substantially. In this series of tests the body (initially a sphere at rest) is subjected to a constant forward acceleration $a$ as its cross-flow dimension is reduced at constant rate $V=\mathrm{d} D / \mathrm{d} t$. Thus the body shape deforms to that of a prolate spheroid with constant axial dimension as it accelerates and the balance of these two parameters (body acceleration versus body shrinking rate) will determine the fluid response. Note that while this test is closely related to the self-propelled scenario, this is still a towed test, involving no propulsive jet.

Using the fact that $D_{o}=L$, and that the acceleration $a$ and velocity of shrinking $V$ are constant, we obtain the following expression for the added-mass term:

$$
\frac{\mathrm{d}}{\mathrm{d} t}\left(m_{a} u\right)=f_{0}\left[\left(1-t^{*}\right)^{3}-3 t^{*}\left(1-t^{*}\right)^{2}\right],
$$

where $t^{*}=t|V| / L=1-D(t) / L$, and $f_{0}=(\pi / 12) \rho D_{o}^{3} a$. This equation is plotted in figure 5 along with the instantaneous added-mass force, $m_{a} a$, that would act on a similar body with constant shape (i.e. which has the same dimensions as the instantaneous dimensions of the shrinking body), without accounting for the term attributable to the change in added mass - we will call this the 'frozen' added-mass term. Note that the energy required to accelerate the body up to a certain speed $u=a t$ is equal to the area under the total, rather than the frozen-added-mass term. As $t^{*} \rightarrow 1$ this energy goes to zero; hence, the net energy spent in the fluid to accelerate a shrinking object in a potential flow goes to zero when the final shape has zero radial dimension.

This implies that the energy imparted to the fluid in the early stage of the manoeuvre is recovered in the final stage, within potential flow theory. As demonstrated in the previous tests, however, forces in a viscous flow can differ from this potential flow prediction because of flow separation effects. Dimensional analysis shows that the governing parameter, the shape change number $\Xi$, is

$$
\Xi=\frac{V^{2}}{a L},
$$

which compares the acceleration imparted to the body with the velocity of shape change divided by the length. Figure 5 shows the pressure force as a function of time for a set of simulations at five different values of the shape change number $\Xi$. As the 


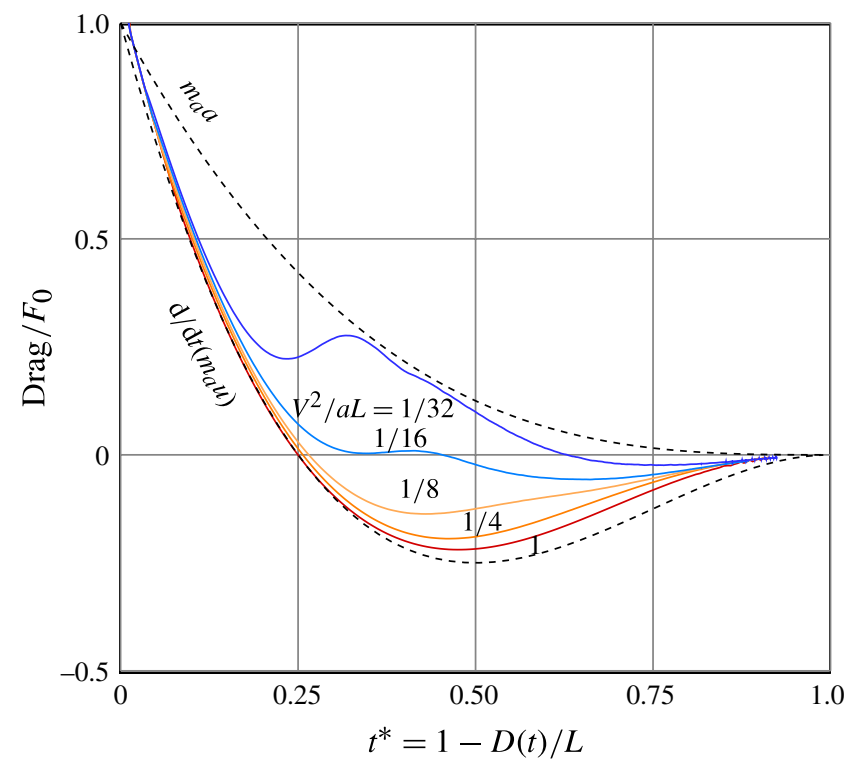

FIGURE 5. (Colour online) Axial fluid force as function of non-dimensional time $t^{*}=$ $1-D(t) / L$ and the shape change number $\Xi=V^{2} / a L$. The dashed black lines are the potential flow predictions and the solid lines are the pressure results in a viscous fluid for the prolate ellipsoids.

value of $\Xi$ is decreased, the plot of the force as function of time moves further away from the potential flow line, implying significant drag effects.

As a reference condition, in the case of a constant-shape body when $x / L \approx 1$ significant flow separation commences; the non-dimensional time for a shrinking body, corresponding to this point in its trajectory, is:

$$
\left.t^{*}\right|_{x=L}=|V| \sqrt{\frac{2}{a L}}=\sqrt{2 \Xi} .
$$

For the lowest value of $\Xi=1 / 32$ (slow deflation), there is a clear bump in the force starting at $t^{*} \approx 0.25=\sqrt{2 \Xi}$, in agreement with the prediction of (4.4), due to significant flow separation. Still, even for this case, it is clear that the shrinking of the body reduces drag, as shown by the decreased fluid force compared to the 'frozen' added-mass force.

Figure 6 shows the normal stress on the surface of the ellipsoid as it shrinks and accelerates for $\Xi=1 / 4$ and $\Xi=1 / 16$. As seen in figure $6(a, b)$, the stress magnitude has been scaled by $\Xi$ such that the initial values are the same for each case.

In figure $6(c-h)$ both cases show that shrinking results in a suction on the top of the spheroid, causing the low-pressure zone to move forward. For $\Xi=1 / 4$, the low-pressure zone moves forward as function of time and is eventually located, almost entirely, on the front half of the body, hence resulting in significant thrust that cancels the drag induced by the high pressure around the stagnation point at the nose. For $t^{*} \geqslant 0.5$, this moving forward is clearly shown, while a high-pressure zone also forms on the trailing section, providing further thrust. This distribution of pressure means that despite experiencing forward acceleration, the flow exerts a thrust force on the body, instead of the expected reactive and drag forces, a highly non-intuitive result. 
(a)

$\Xi=1 / 4$

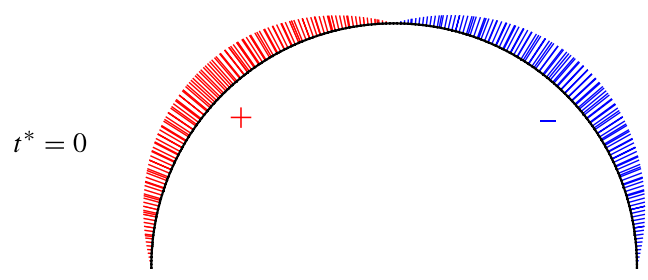

(c)

$t^{*}=1 / 4$

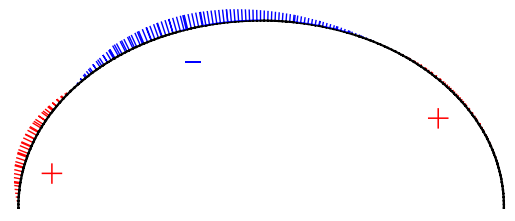

(e)

$t^{*}=1 / 2$

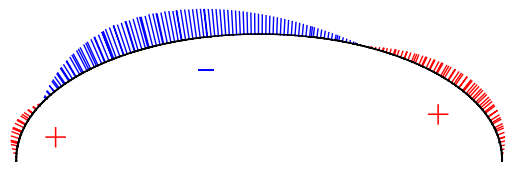

$(g)$

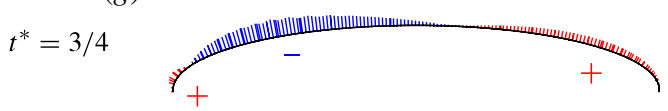

(b)

$\Xi=1 / 16$

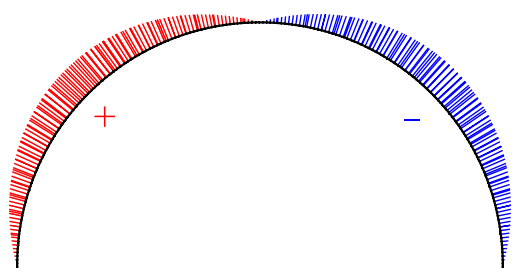

(d)

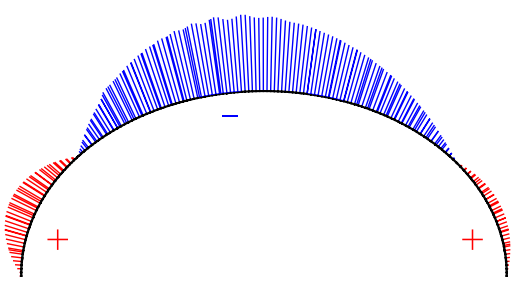

$(f)$

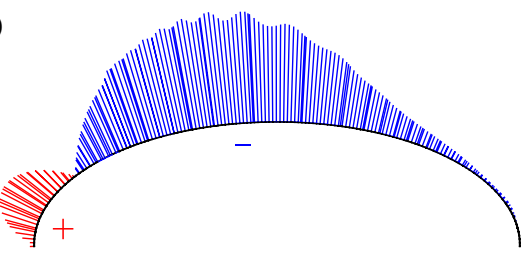

(h)

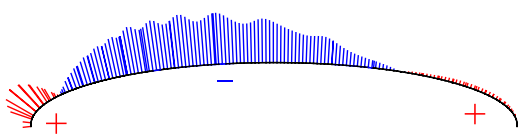

FIgURE 6. (Colour online) Normal stress on the surface of a simultaneously deforming and accelerating prolate ellipsoid for two shape-change numbers, $\Xi=V^{2} / a L=1 / 4$ (left-hand column) and $1 / 16$ (right-hand column), as a function of non-dimensional time $t^{*}=1-D(t) / L$ (from 0 to 3/4, top to bottom). The body is accelerating right-to-left. Regions of high: $(+)$, and low (-) pressure are labelled. The stress magnitudes are scaled by $\Xi$ to equalize the initial force for these two cases and by $r=\sqrt{y^{2}+z^{2}}$ to graphically represent the stress integral around the axis of revolution at each point. This figure is presented in an axisymmetric style to aid visualization; the simulations are fully three-dimensional.

For $\Xi=1 / 16$ the favourable pressure on the trailing section is mostly lost due to the onset of boundary layer separation, but there is still a small net thrust due to suction on the forward half of the body, as the suction pressure also moves towards the front as function of time.

\section{Escape manoeuvre of a self-propelled deflating body}

In this section we consider a self-propelled rocket, focusing on an initially spherical body which deflates from a spherical down to a streamlined prolate-spheroid shape, and compare this to other rocket configurations.

The results of the previous section showed that we should use the largest possible value of the shape-change number $\Xi$ for our shape-changing rocket. However, there 
are limits to the achievable $\Xi$ value, and simply increasing the rate at which the diameter is reduced does not change $\Xi$ significantly. In fact, the primary factor controlling the value of $\Xi$ in a self-propelled body is the area of the jet aperture $A_{j}$. Consider the equation of motion of the spheroid rocket when $u=0$ and therefore $f_{d}=0$. Then (2.3) can be simplified as:

$$
\left(m+m_{a}\right) a=-U_{j} \dot{m} .
$$

Since $m=\pi \rho L D^{2} / 6$ for a spheroid, we have:

$$
\dot{m}=\frac{1}{3} \pi \rho L D V=-\rho U_{j} A_{j} .
$$

Using $D=D_{o}=L$, (2.4) simplifies to $m_{a}=m / 2$ for the initially spherical neutrally buoyant shape and we find:

$$
\Xi_{o}=\frac{9}{16} \frac{A_{j}}{A_{o}},
$$

where $A_{o}=\pi L^{2} / 4$ is the initial cross-stream area of the rocket.

Equation (5.3) shows that there is a limiting relation of $\Xi<9 / 16$ for a selfpropelled sphere, and that increasing $A_{j}$ increases $\Xi_{o}$. However, a constant large aperture would limit the final value of the lateral dimension of the spheroid (at the end of the shrinking process) to a value larger than or equal to the opening of the aperture. This constrains the ability to streamline the object and hence the amount of recoverable kinetic energy. Therefore, an aperture which starts large and shrinks with the body will enable the largest value of $\Xi$ and allow a streamlined final shape, optimizing the energy recovery and reducing separation to maximize the rocket acceleration and total distance travelled.

In our tests we chose $A_{j}=\pi L D / 100$, which allows a constant shrinking rate $V$ to produce a constant jet velocity $U_{j}$ as shown by (5.2). The relatively small size of the jet $\left(A_{j}=A_{o} / 25\right)$ was chosen to ensure the final form was streamlined and to demonstrate the robust drag-reducing ability of the shrinking spheroid rocket even with small values of $\Xi$.

We proceed then to study an initially spherical body at rest, which subsequently shrinks its cross-flow dimension, while preserving its in-line length, to expel the contained fluid and propel itself. The jet is ramped up to a constant velocity $U_{j}$ after a very short time, chosen here to be when only $4 \%$ of the fluid propellant has been ejected; and continues jetting until a small amount of mass remains, chosen again to be $4 \%$ of the initial mass which corresponds to a $5: 1$ prolate shape. The final streamlined shape is chosen to be a 5:1 prolate spheroid as this is close to the optimal value that balances the pressure and viscous drag for a given volume payload to optimize overall drag (Hoerner 1965). This is a choice made to focus the study; results would apply to similar shapes as well.

The body is allowed to accelerate in the thrust direction, with the other five translational and rotational degrees of freedom fixed to zero values. Using the mass flux of the jet and integrating the fluid contact forces on the body, the body motion equation is integrated in time to update the body's position and velocity. The Reynolds number based on the jet velocity for the simulations is $R e_{j} \equiv U_{j} L / v=10000$.

Figure 7 shows snapshots of the simulation results for the shrinking spheroid, labelled in terms of the distance travelled at each time. As shown in the previous section, the recovery of fluid kinetic energy that provides forward thrust is reduced if the flow is separated, because then vorticity is shed in the fluid together with some 
(a)

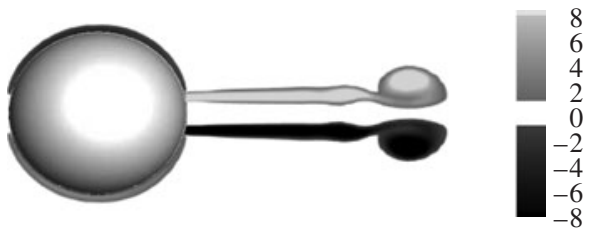

(b)

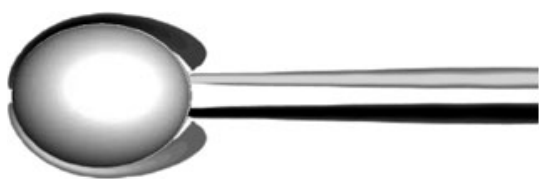

(c)

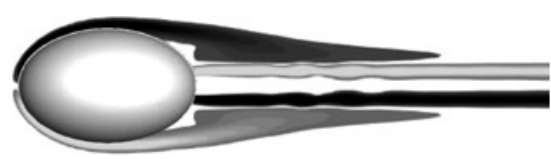

(d)

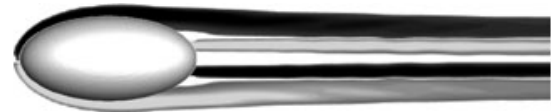

(e)

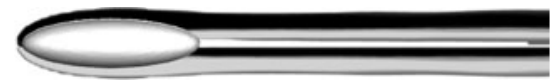

FIGURE 7. Fast escape of a prolate-spheroid rocket shown in a body-fixed frame. Contours of vorticity are shown on a plane passing through the body's longitudinal axis of symmetry. The simulations are fully three-dimensional; the body is accelerating right-to-left. Despite travelling many body lengths with a relatively bluff shape, the flow around the rocket does not separate noticeably, enabling both reduced drag forces and recovery of the fluid kinetic energy, hence producing useful thrust. (a) $x=0.2 L ;(b) x=1.0 L ;(c) x=3.5 L ;(d) x=8.0 L$; (e) $x=16.0 L$.

entrained energy that is lost to the flow. In addition, the shed vortices would also increase the drag on the body, as shown in figure $4(b)$.

It is remarkable, however, as shown in figure 7 , that the self-propelled rocket does not experience noticeable separation, despite both its initially bluff shape, and having travelled many body lengths. This is because the rate of collapse induces a boundary velocity which is approximately of the same magnitude as the body speed during the initial, bluff-body phase. As the rocket comes up to speed, the shape is made progressively more streamlined. The overall effect is that the collapse prevents separation, hence keeping the drag force very small. This in turn enables the addedmass energy recovery, compounding the drag reduction effect.

We next compare this cephalopod-inspired configuration to three other jet-propelled test cases:

(a) a rigid spherical rocket;

(b) a shrinking spherical rocket; and

(c) a rigid 5:1 streamlined rocket, which is similar to a streamlined torpedo.

The size of the torpedo is set to match the initial volume of the other cases, thereby giving it the same amount of 'fuel' to expel. The mass of the bodies as a function of time and the mass expelled at each instant are identical in all cases. As in $\$ 2.2$, this implies that the rigid rockets empty of their fluid propellant such that the final mass is $m_{f}$. For example, this is accomplished in water rockets by filling the void with a low-density gas. By using the same mass and propulsive jet force for all cases, we ensure that the body shape is the only discriminating factor. We did not include the analysis of a shrinking prolate spheroid with a constant 5:1 aspect ratio, because it does not exhibit significant separation due to its streamlined shape, while its added 

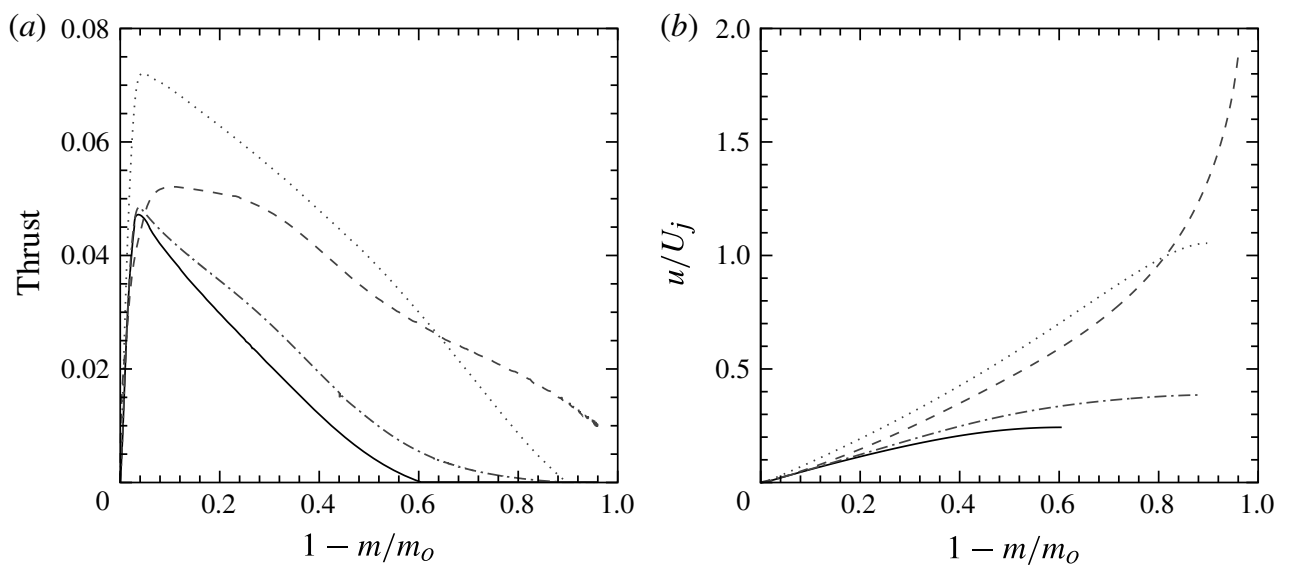

FIGURE 8. (a) Net thrust coefficient profiles as a function of the relative amount of mass ejected $\left(\left(m_{o}-m\right) / m_{o}\right)$. The four cases are: - , a rigid spherical rocket; - - - a collapsing spherical rocket; ...., a rigid 5:1 prolate spheroid; and -- , a spheroid deflating radially from a spherical shape to a 5:1 shape. The net force is the jet thrust force minus the integrated normal and tangential stresses on the body and is scaled by the jet velocity and the initial spherical cross-sectional area $A_{o}=\pi L^{2} / 4$. (b) The body velocity profiles for the same cases as in $(a)$, scaled by the jet velocity.

mass is small (one fifth of a sphere), and hence does not demonstrate the effects of energy recovery either.

Figure 8 shows the thrust force and the escape velocity for the model problems as a function of the ejected mass ratio $\left(m_{o}-m\right) / m_{o}$. The rigid spherical rocket shows the worst performance, as expected, with a maximum achieved velocity of around $25 \%$ of the jet velocity. The shrinking spherical case shows an increased performance, accelerating up to around $40 \%$ of the jet velocity. Comparing these to the potential flow values in (2.5) and (2.6) we see that the flow separation and resulting drag in a viscous fluid have significantly reduced the achievable speed-up of the sphere rocket. The rigid torpedo shows improved performance because of its streamlined shape, relative to the spherical cases. This reduces the drag and increases the net thrust throughout the acceleration phase, enabling it to achieve a peak velocity of $106 \%$ of the jet velocity.

By comparison, the model case of a shrinking streamlining spheroid starts as a bluff body, and therefore has a lower initial acceleration than the torpedo. However, the gradual streamlining and size reduction pay off in the end through the hydrodynamic mechanisms described above, enabling the rocket to achieve a peak velocity of $190 \%$ of the jet velocity. This is an $80 \%$ increase in speed relative to the rigid streamlined torpedo, therefore imparting $320 \%$ of the kinetic energy to the final mass $m_{f}$. This demonstrates that the benefits of size reduction are enormous, even for this shapechange number, which is smaller than optimal.

Figure 9 compares the net thrust force on the body (the jet thrust minus the integrated normal and tangential stresses) to the added-mass forces. We decompose the added-mass force as given in (2.3) into a 'frozen' part, $m_{a} a$, and a deflating-body additional added-mass term, $\dot{m}_{a} u$. Note that the latter is more than $50 \%$ of the net thrust in the second half of the acceleration phase. By starting the simulation with the body having the form of a sphere, which is a non-streamlined object, and then 


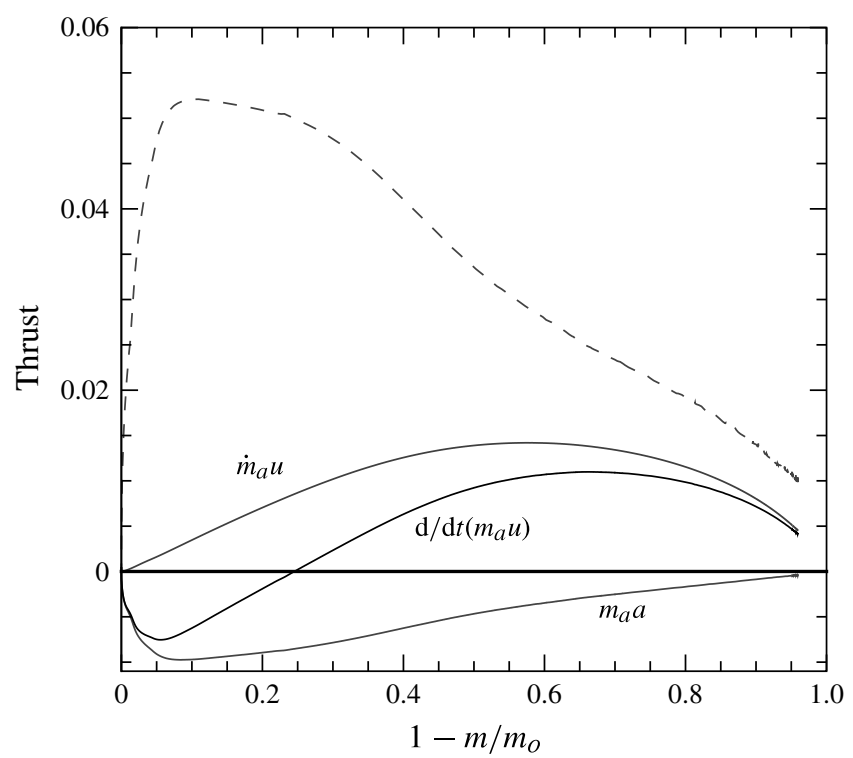

FIGURE 9. Net thrust coefficient analysis for the deflating prolate spheroid. The dashed line is the predicted net thrust force, while the solid lines are the unsteady added-mass term and its frozen $\left(m_{a} a\right)$ and dynamic $\left(\dot{m}_{a} u\right)$ components. Forces scaled as in figure 8.

having it collapse gradually into a streamlined shape, in a process closely resembling that reported for the cephalopods, the spheroid rocket is able to store energy in the fluid in the initial acceleration and then reclaim that energy as thrust when the mass is lower, increasing its effectiveness. Therefore, while a streamlined body with reduced cross-section is effective in reducing drag, the dynamic effect related to the added mass is what enables the collapsing spheroid, through a late blast of acceleration, to achieve nearly double the escape speed relative to the rigid torpedo shape.

\section{Discussion and conclusions}

We show through analytic arguments and numerical simulation that a deformable body immersed in a dense fluid may recover a large portion of the fluid added mass via size reduction and use this to achieve impressive propulsive velocities. As an initial result, we show that the analytic equations governing a deflating spherical rocket in potential flow result in rocket speeds faster than the same rocket in a vacuum. Hence, the added-mass force acts overall not to hinder the rocket's acceleration (as it does for a rigid body) but to amplify it due to recovery of the kinetic energy conveyed initially to the fluid as the body shrinks.

We next extend this result to a viscous fluid case, employing numerical simulation of the Navier-Stokes equations to model a rocket that expels mass and produces a propulsive jet by collapsing its lateral dimension, changing from a bluff to a streamlined shape. For high ratios of expelled mass to initial mass, this shape achieves nearly double the speed relative to an optimally streamlined, rigid-hull, jet-propelled rocket.

We identify the basic mechanisms that allow this ultra-fast escape.

(a) The near-elimination of separation even in the early phases, when the body shape is bluff, for appropriate values of the velocity of body shrinking, $V$, relative to its 
acceleration, $a$, as expressed by the non-dimensional quantity $\Xi=V^{2} /(a L)$, where $L$ the lateral dimension of the body.

(b) The recovery of added-mass energy as the body deflates. This, however, depends critically on the lack of flow separation, ensured by the first mechanism.

(c) Employing an optimal energy utilization, whereby the body transfers energy to the fluid at the onset of the manoeuvre and then recovers it in the form of thrust force, through the combination of the two previous mechanisms, at the later stages of the motion, when its mass is reduced and the effects of the thrust on acceleration are maximized.

All mechanisms are critically important and must be combined to achieve high performance: the energy recovery, for which an upper estimate can be obtained using potential flow, can lead to considerable thrust augmentation, adding a component equal to more than $90 \%$ of the jet-generated thrust near the end of the manoeuvre. The elimination of separation, especially when the body is in the first half of the manoeuvre and its shape is basically bluff, is critical to achieving fluid energy recovery. Finally, 'storing' kinetic energy in the fluid in the early part of the manoeuvre allows its recovery at the later part of the trajectory when it is most efficiently used.

The inspiration for the present work came from the reported swimming abilities of cephalopods, which achieve high performance despite their lack of a skeleton. This led to the present investigation, but we have simplified the problem considerably, focusing basically on a model of the mantle of a squid or octopus, omitting the influence of other parts of the body of the animal, such as the legs and fins, as well as the mechanics of actuation. The total expelled mass of an animal is limited, and hence it cannot achieve the very high speeds that are achievable only when the expelled mass is practically equal to the initial mass. Also, we possibly exaggerated the initial hyperinflation employed by animals, modelling the resulting shape as a sphere. As a result, one cannot draw conclusions on the details of the animal performance; however, the mechanisms we identified can clearly be in use by the animals and can fully explain the use of such manoeuvres for fast escapes.

The final, length-based Reynolds number for the prolate shrinking rocket is around 20000 . While this falls nicely within the values of cephalopod jetting described in the introduction, those values range from $10^{3}-10^{5}$. We found that tests run at a lower Reynolds number of $R e_{j}=1000$ showed a decrease in performance due to the increased role of viscous drag on the streamlined prolate shape, but that this also affects all the other cases, including the torpedo test case; hence, qualitatively, the result holds true. At higher $R e$, we might expect slightly improved performance, though this would depend on the continued successful inhibition of separation at the higher speed.

In this work we focused on the external flow, and have avoided specifying the details and energetics of the shrinking mechanism. This is a lengthy and somewhat separate topic which will feature large in forthcoming work. Our model for the propelling jet flow was also greatly simplified, prescribing a constant-magnitude uniform-profile jet and not solving for the internal cavity flow. Hence no attention was paid to optimizing the jet flow, an important problem in itself (Linden \& Turner 2004; Anderson \& Grosenbaugh 2005; Bartol et al. 2009; Moslemi \& Krueger 2011). Also, there is a connection of the present work to the hydrodynamics of medusae (Dabiri, Colin \& Costello 2006), particularly when the mode of 'umbrella' deflation 
is assumed; for this problem, the interaction of the flow around the body with the jet flow is significant, and could provide further, potentially beneficial, effects.

Finally, there are some parallels that can be drawn between mechanisms studied in this paper and the mechanisms employed by fast-starting fish, which bend their bodies to a C- or S-shape at the onset of the manoeuvre, creating large curvatures of their backbone, and then straighten it to generate the forces that power the manoeuvre (Domenici \& Blake 1997). As shown in Gazzola et al. (2012), the initial large curvature of the $\mathrm{C}$-shape manoeuvre serves to impart kinetic energy to as large a mass of water around the body as possible; this kinetic energy, combined with body-shed vorticity, is entrained and forms the large vortical patterns that propel the body to achieve impressive acceleration. This is conceptually similar to the mechanism of imparting kinetic energy to a large mass of the fluid in the early phase of the manoeuvre, and then recovering it in the later phase, as we showed in the fast-start manoeuvre of a cephalopod-like body. In the case of the fish, this energy ends up in the propulsive pair of vortices which serve to accelerate the body forward. Finally, the unsteady motion of the fast-starting fish is tightly monitored so that no uncontrolled vorticity is shed to generate undesirable drag forces (Gazzola et al. 2012), also in analogy to the separation elimination that we show herein.

Overall this work demonstrates that a cephalopod-like deformable body can vastly outperform the fast-start escape of a rigid jet-propelled vehicle, despite the fact that the latter is better streamlined. The study therefore serves as another example of viscous flow control under highly unsteady conditions to optimize locomotion.

\section{Acknowledgements}

The authors wish to acknowledge support from the Singapore-MIT Alliance for Research and Technology through the CENSAM Program, and from the MIT Sea Grant Program.

\section{REFERENCES}

Anderson, E. J. \& Grosenbaugh, M. A. 2005 Jet flow in steadily swimming adult squid. J. Expl Biol. 208, 1125-1146.

Anderson, E. J., Wuinn, W \& DEMont, M. E. 2001 Hydrodynamics of locomotion in the squid Loligo pealei. J. Fluid Mech. 436, 249-266.

Bartol, I. K., Krueger, P. S., Thompson, J. T. \& Stewart, W. J. 2009 Pulsed jet dynamics of squid hatchlings at intermediate Reynolds numbers. J. Expl Biol. 212, 1506-1518.

Childress, S., Spagnolie, S. E. \& Tokieda, T. 2011 A bug on a raft: recoil locomotion in a viscous fluid. J. Fluid Mech. 669, 527-556.

Childress, S., Vanderberghe, N. \& Zhang, J. 2006 Hovering of a passive body in an oscillating airflow. Phys. Fluids 18, 117103.

Dabiri, J. O., Colin, S. P. \& Costello, J. H. 2006 Fast-swimming hydromedusae exploit velar kinematics to form an optimal vortex wake. J. Expl Biol. 209, 2025-2033.

DANiel, T. L. 1984 Unsteady aspects of aquatic locomotion. Am. Zool. 24 (1), 121-134.

Domenici, P., Blagburn, J. M. \& Bacon, J. P. 2011 a Animal escapology I: theoretical issues and emerging trends in escape trajectories. J. Expl Biol. 214, 2463-2473.

Domenici, P., Blagburn, J. M. \& BACON, J. P. $2011 b$ Animal escapology II: escape trajectory case studies. J. Expl Biol. 214, 2474-2494.

Domenici, P. \& BLAKe, R. 1997 The kinematics and performance of fish fast-start swimming. J. Expl Biol. 200, 1165-1178.

Forsythe, J. W. \& HANLON, R. T. 1988 Behavior body patterning and reproductive biology of Octopus bimaculoides from California USA. Malacologia 29, 41-55. 
Gazzola, M., van Rees, W. M. \& Koumoutsakos, P. 2012 C-start: optimal start of larval fish. J. Fluid Mech. 698, 5-17.

Gosline, J. M. \& DeMont, M. E. 1985 Jet-propelled swimming in squid. Sci. Am. 252, 96-103.

Hoerner, S. 1965Fluid Dynamic Drag. Published by the author, Hoerner Fludi Dynamics, Bricktown, New Jersey.

HuFFARD, C. L. 2006 Locomotion by Abdopus aculeatus (Cephalopoda: Octopodidae): walking the line between primary and secondary defenses. J. Expl Biol. 209, 3697-3707.

Kanso, E., Marsden, J. E., Rowley, C. W. \& Melli-Huber, J. B. 2005 Locomotion of articulated bodies in a perfect fluid. J. Nonlinear Sci. 15, 255-289.

Linden, P. F. \& TURNER, J. S. 2004 Optimal vortex rings and aquatic propulsion mechanisms. Proc. R. Soc. Lond. B 271, 647-653.

Margolin, L. G., Rider, W. J. \& Grinstein, F. F. 2006 Modeling turbulent flow with implicit LES. J Turbul 7, 1-27.

Moslemi, A. \& Krueger, P. S. 2011 The effect of Reynolds number on the propulsive efficiency of a biomorphic pulsed-jet underwater vehicle. Bioinsp. Biomim. 6, 026001.

Neumeister, H., Ripley, B., Preuss, T. \& Gilly, W. F. 2000 Effects of remperature on escape jetting in the squid Loligo opalescens. J. Expl Biol. 203, 547-557.

PACKARD, A. 1969 Jet propulsion and the giant fibre response of Loligo. Nature 221, 875-877.

SAFFMAN, P. G. 1967 Self-propulsion of a deformable body in a perfect fluid. J. Fluid Mech. 28, 385-389.

Spagnolie, S. E. \& Shelley, M. J. 2009 Shapechanging bodies in fluid: hovering, ratcheting, and bursting. Phys. Fluids 21, 013103.

Wells, M. J. 1990 Oxygen extraction and jet propulsion in Cephalopods. Can. J. Zool. 68, $815-824$.

Weymouth, G. D., Dommermuth, D. G., Hendrickson, K. \& Yue, D. K.-P. 2006 Advancements in Cartesian-grid methods for computational ship hydrodynamics. 26th Symposium on Naval Hydrodynamics, Rome, Italy, 17-22 September 2006.

Weymouth, G. D. \& Triantafyllou, M. S. 2012 Global vorticity shedding for a shrinking cylinder. J. Fluid Mech. 702, 470-487.

Weymouth, G. D. \& Yue, D. K.-P. 2011 Boundary data immersion method for Cartesian-grid simulations of fluid-body interaction problems. J. Comput. Phys. 230, 16.

Wibawa, M. S., Steele, S. C., Dahl, J. D., Rival, D. E., Weymouth, G. D. \& TRIANTAFYlloU, M. S. 2012 Global vorticity shedding for a vanishing wing. J. Fluid Mech. 695, 112-134.

Williamson, G. R. 1965 Underwater observations of the squid Illex illecebrosus Lesueur in Newfoundland waters. Can. Field Natur. 79, 239-247. 